Tanaka, et al. • page S1

\title{
Enantioselective Total Synthesis of Ustiloxin D
}

Hiroko Tanaka, Andrew M. Sawayama, and Thomas J. Wandless

Department of Chemistry, Stanford University, Stanford, CA 94305 


\section{Supporting Information}

Proton magnetic resonance spectra $\left({ }^{1} \mathrm{H}\right.$ NMR) were recorded on Varian UI-500 (500 $\mathrm{MHz}$ ) spectrometer using $\mathrm{D}_{2} \mathrm{O}$ as solvent. Chemical shifts are reported in ppm from tetramethylsilane using the water resonance as an internal standard (4.67 ppm). Data are reported as follows: chemical shift, multiplicity $(\mathrm{s}=$ singlet, $\mathrm{d}=$ doublet, $\mathrm{t}=$ triplet, $\mathrm{q}=$ quartet, $\mathrm{br}=$ broadened, mult $=$ multiplet $)$, coupling constants $(\mathrm{Hz}) .{ }^{13} \mathrm{C}$ NMR spectra were recorded with complete proton decoupling on a Varian UI-500 (125.5 MHz) spectrometer. Chemical shifts are reported in ppm from tetramethylsilane using a trace of 1,4-dioxane $(66.5 \mathrm{ppm})$ as an internal standard.

HPLC traces were obtained using a Beckman System Gold 508 Autosampler, 125S Solvent Module and 166NM Single Wavelength Detector with a Waters Xterra M8 phenyl $5 \mu \mathrm{m}$ column $(4.6 \times 100 \mathrm{~mm}) .98 \%$ water and $2 \%$ acetonitrile was used as eluent, the water containing $0.05 \%$ trifluoroacetic acid. The Pd-mediated etherification formed two diastereomers (major is desired) that were carried through subsequent steps. Thus, the final synthetic step produced a 2:1 mixture of ustiloxin D and C2-epi-ustiloxin D. The 2:1 mixture of ustiloxin isomers were separated by preparative HPLC using a Waters Delta 600 Controller and 2996 Photodiode Array Detector with a Waters Xterra M8 phenyl $5 \mu \mathrm{m}(19 \times 100 \mathrm{~mm}) .95 \%$ water and 5\% acetonitrile was used as eluent, the water containing $0.05 \%$ trifluoroacetic acid. Products were detected at $220 \mathrm{~nm}$. The specific rotation of Ustiloxin D was determined using a Jasco DIP-1000 digital polarimeter. 


\section{Comparison of synthetic and natural ustiloxin D using ${ }^{1} \mathrm{H}$ and ${ }^{13} \mathrm{C}$ NMR}

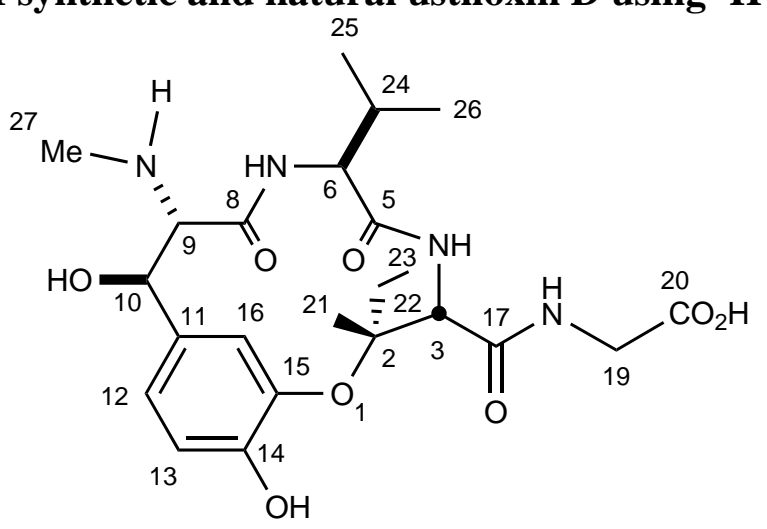

\begin{tabular}{|c|c|c|c|c|c|}
\hline \multicolumn{3}{|c|}{${ }^{1}$ H NMR $\delta$ (ppm) } & \multicolumn{3}{|c|}{${ }^{13}$ C NMR $\delta$ (ppm) } \\
\hline $\begin{array}{c}\text { Position } \\
3^{2}\end{array}$ & Synthetic & Natural & $\begin{array}{c}\text { Position } \\
2\end{array}$ & $\begin{array}{c}\text { Synthetic } \\
86.0\end{array}$ & $\begin{array}{c}\text { Natural }^{1} \\
85.8\end{array}$ \\
\hline 6 & $3.90(\mathrm{~d}, \mathrm{~J}=10)$ & $3.89(\mathrm{~d}, \mathrm{~J}=10)$ & 3 & 59.5 & 59.3 \\
\hline $9^{*}$ & $3.74(\mathrm{~d}, \mathrm{~J}=9)$ & $3.67(\mathrm{~d}, \mathrm{~J}=9)$ & 5 & 171.2 & 171.5 \\
\hline 10 & $4.60(\mathrm{~d}, \mathrm{~J}=9.5)$ & $4.57(\mathrm{~d}, \mathrm{~J}=9.5)$ & 6 & 60.2 & 60.2 \\
\hline 12 & $7.01(\mathrm{dd}, \mathrm{J}=2,8.5)$ & $7.01(\mathrm{dd}, \mathrm{J}=2,8.5)$ & 8 & 166.3 & 166.3 \\
\hline 13 & $6.90(\mathrm{~d}, \mathrm{~J}=8)$ & $6.90(\mathrm{~d}, \mathrm{~J}=8)$ & 9 & 69.0 & 68.9 \\
\hline 16 & $6.84(\mathrm{br}, \mathrm{s})$ & $6.84(\mathrm{br}, \mathrm{s})$ & 10 & 73.1 & 73.0 \\
\hline $19^{\#}$ & $3.67(\mathrm{~s})$ & $3.59(\mathrm{~s})$ & 11 & 130.7 & 130.7 \\
\hline 21 & $1.53(\mathrm{~s})$ & $1.53(\mathrm{~s})$ & 12 & 122.7 & 122.7 \\
\hline \multirow[t]{2}{*}{22} & $1.52(\mathrm{dq}, \mathrm{J}=7.3,21)$ & $1.52(\mathrm{dq}, \mathrm{J}=7.5,21)$ & 13 & 119.1 & 119.1 \\
\hline & $1.97(\mathrm{dq}, \mathrm{J}=7,21)$ & $1.97(\mathrm{dq}, \mathrm{J}=7,21)$ & 14 & 151.0 & 151.0 \\
\hline 23 & $0.91(\mathrm{t}, \mathrm{J}=7.5)$ & $0.91(\mathrm{t}, \mathrm{J}=7.5)$ & 15 & 142.7 & 142.7 \\
\hline 24 & $1.69(\mathrm{~m})$ & $1.69(\mathrm{~m})$ & 16 & 124.0 & 124.0 \\
\hline 25 & $0.67(\mathrm{~d}, \mathrm{~J}=7)$ & $0.67(\mathrm{~d}, \mathrm{~J}=7)$ & 17 & 171.1 & 171.2 \\
\hline 26 & $0.59(\mathrm{~d}, \mathrm{~J}=7)$ & $0.59(\mathrm{~d}, \mathrm{~J}=6.5)$ & 19 & 43.2 & 41.9 \\
\hline \multirow[t]{8}{*}{$27^{\neq}$} & $2.57(\mathrm{~s})$ & $2.52(\mathrm{~s})$ & 20 & 175.3 & 173.5 \\
\hline & & & 21 & 21.3 & 21.4 \\
\hline & & & 22 & 32.4 & 32.3 \\
\hline & & & 23 & 8.1 & 8.0 \\
\hline & & & 24 & 29.1 & 29.1 \\
\hline & & & 25 & 18.5 & 18.4 \\
\hline & & & 26 & 18.1 & 18.0 \\
\hline & & & 27 & 32.4 & 32.4 \\
\hline
\end{tabular}

HRMS calculated for $\mathrm{C}_{23} \mathrm{H}_{34} \mathrm{~N}_{4} \mathrm{O}_{8}(\mathrm{M}+\mathrm{H}): 495.2449$, found (MALDI): 495.2455.

Specific rotation of synthetic ustiloxin D: $[\alpha\rfloor_{D}^{27.5}=-49.7^{\circ}\left(\mathrm{c} 0.35, \mathrm{H}_{2} \mathrm{O}\right)$.

Specific rotation of natural ustiloxin D: $\lfloor\alpha\rfloor_{D}^{20}=-48.8^{\circ}\left(\mathrm{c} 0.5, \mathrm{H}_{2} \mathrm{O}\right)$.

${ }^{1}$ Cao, B.; Park, H.; Joullie, M. M.; J. Am. Chem. Soc. 2002, 124, 520-521.

${ }^{2}$ Known resonance at $\delta 4.77 \mathrm{ppm}$ hidden by $\mathrm{H}_{2} \mathrm{O}$ resonance $(\delta 4.67 \mathrm{ppm})$.

*\#\# $\mathrm{C}-\mathrm{H}$ protons adjacent to $\mathrm{pH}$-sensitive functional groups. 


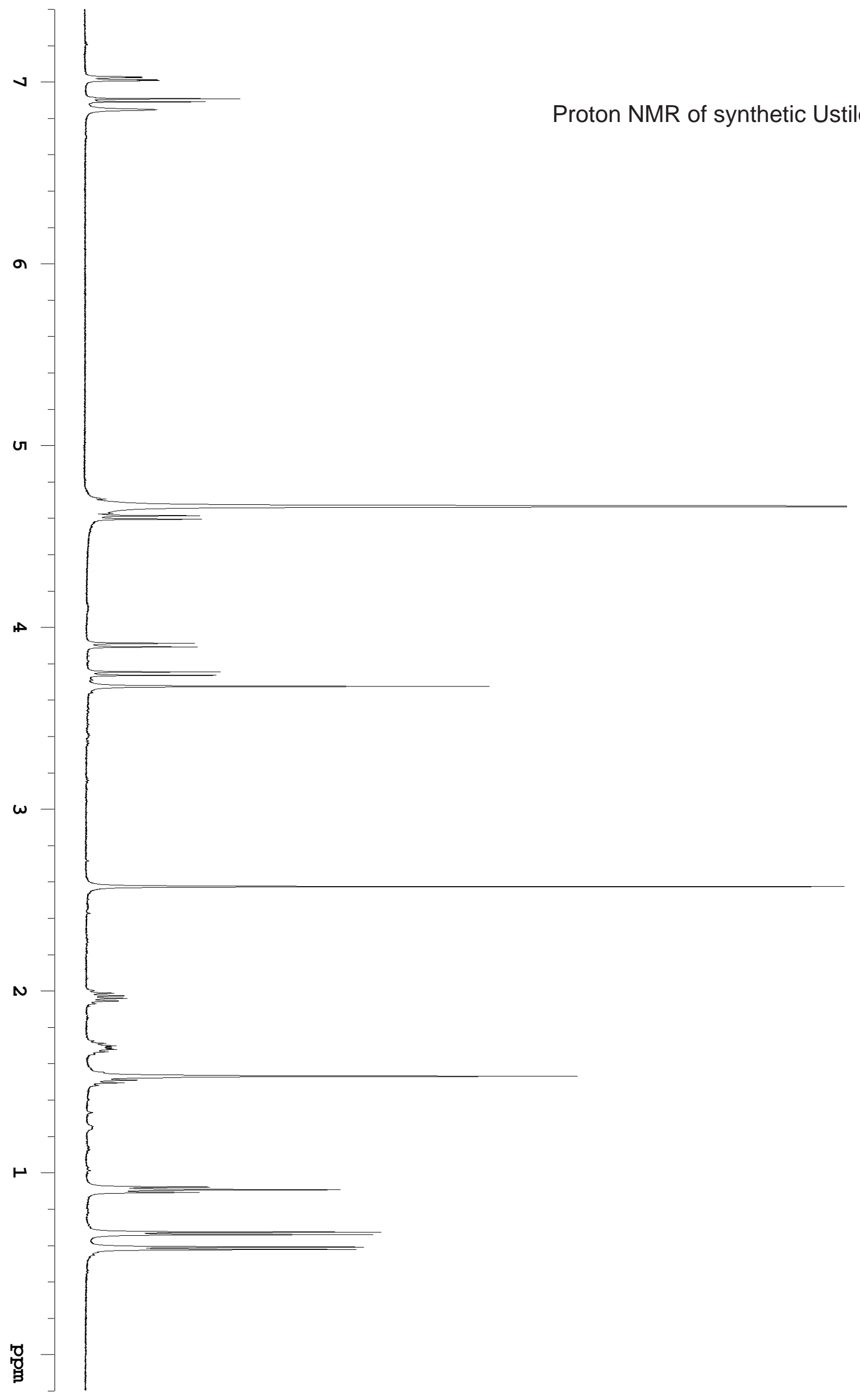




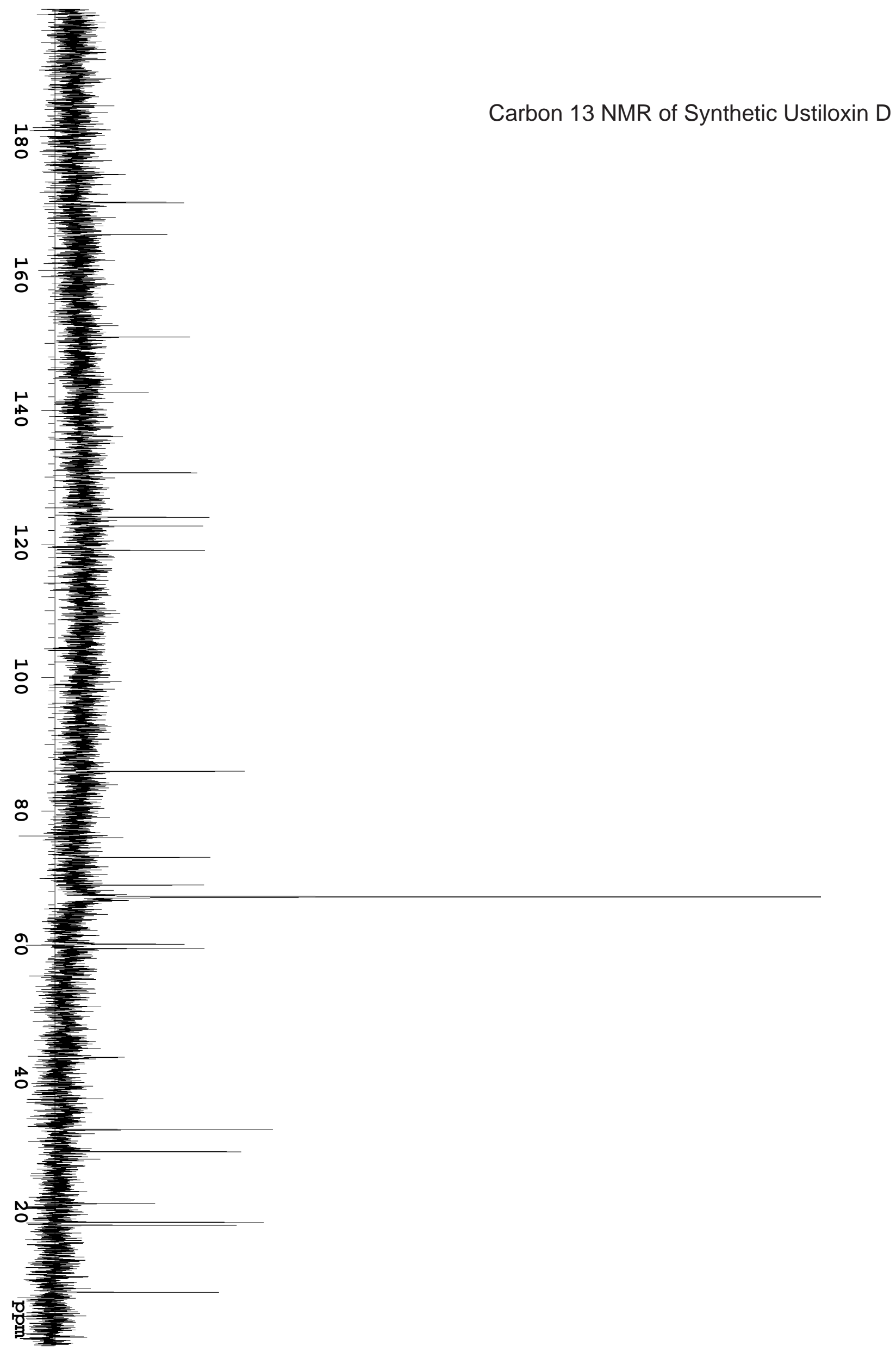




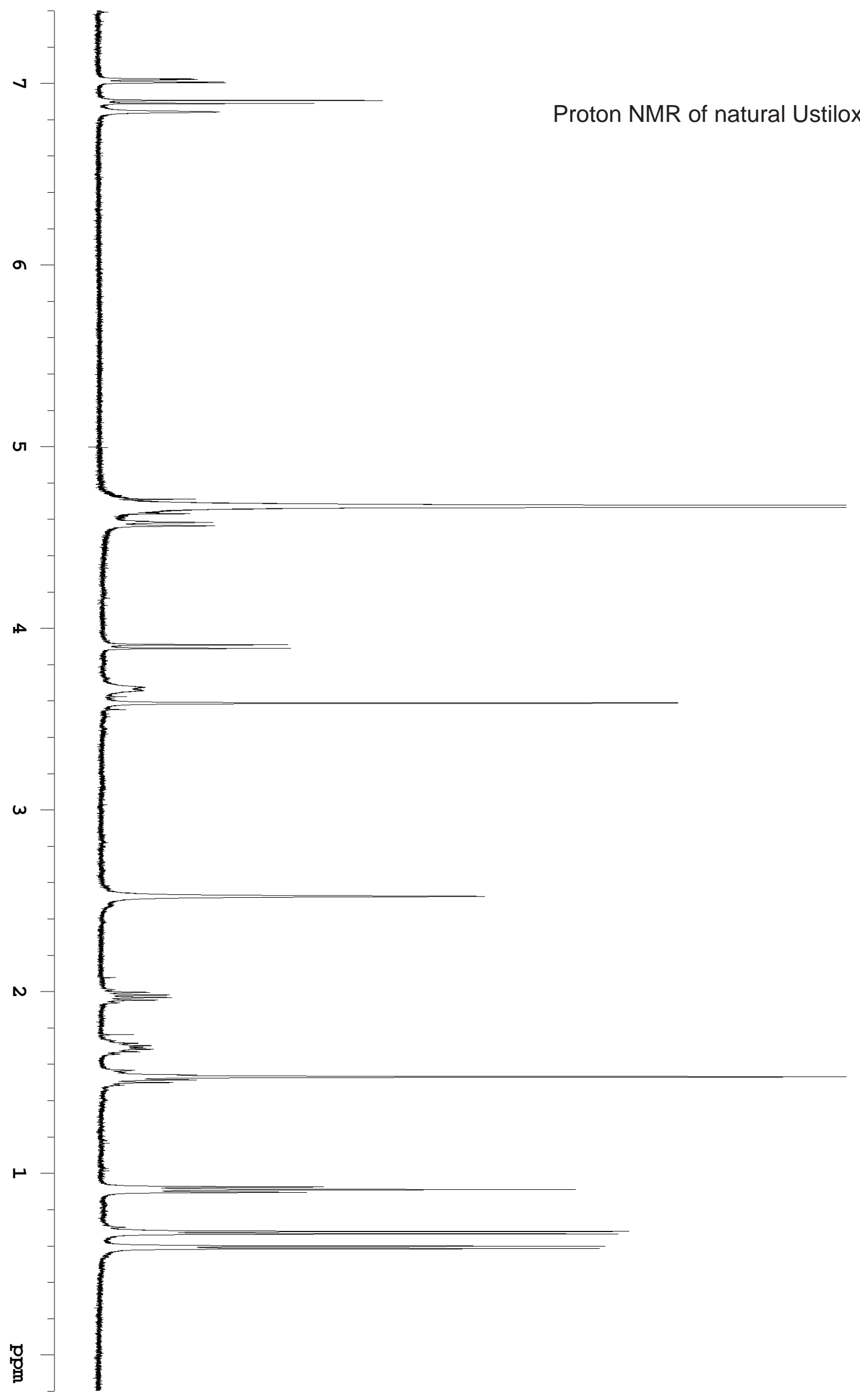




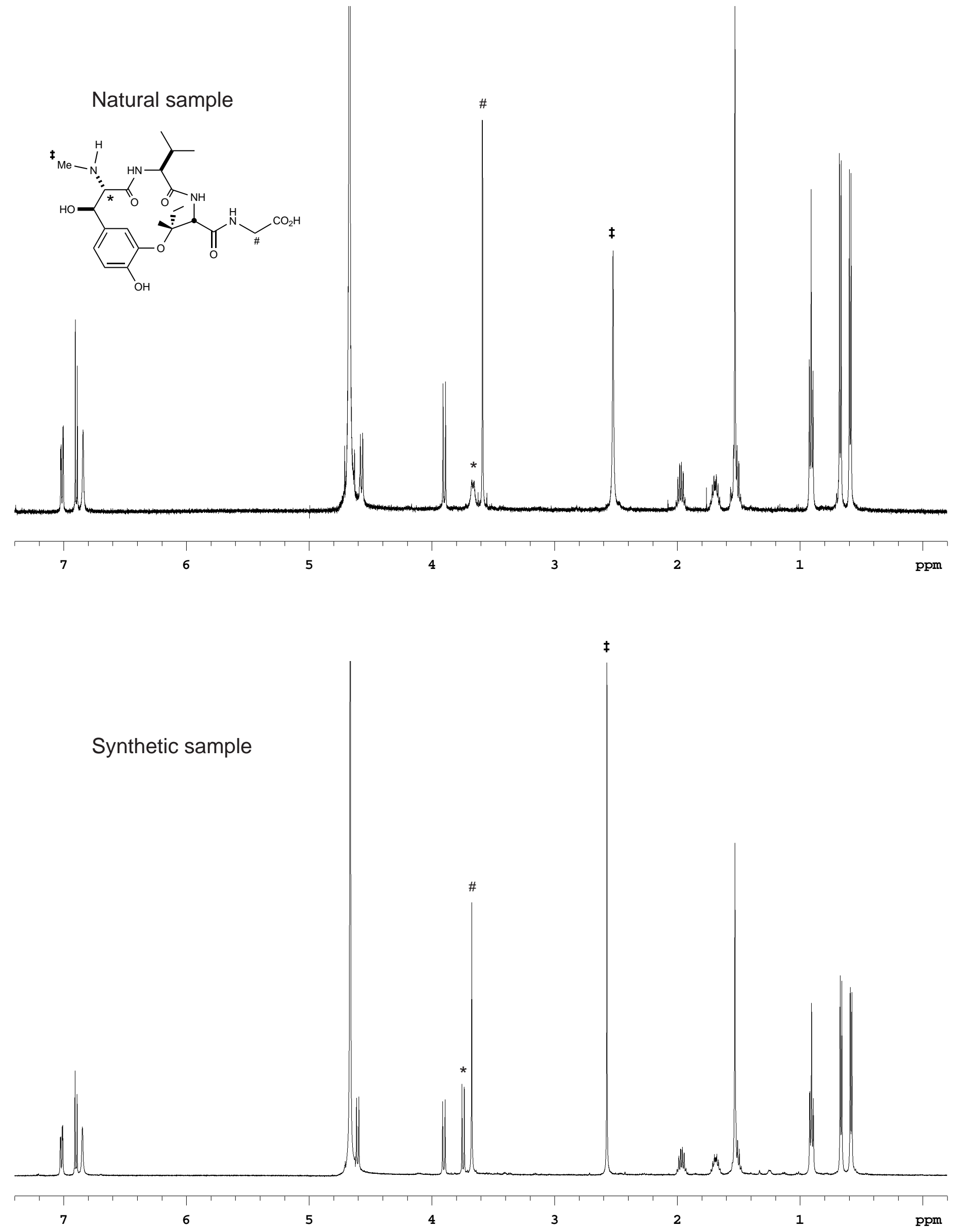


Tanaka et al. • page S8

Natural sample

(0.3 to $2.3 \mathrm{ppm}$ )

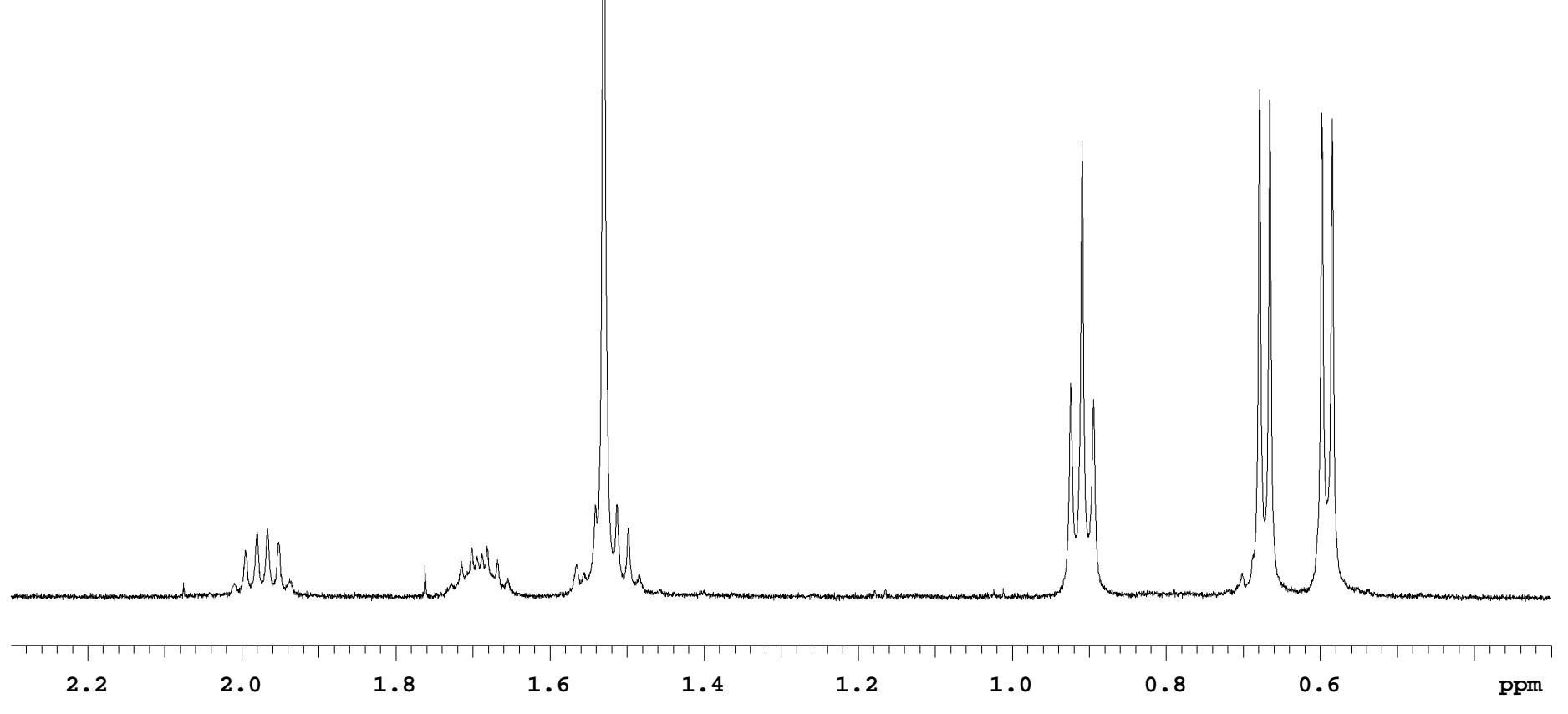

Synthetic sample

(0.3 to $2.3 \mathrm{ppm}$ )

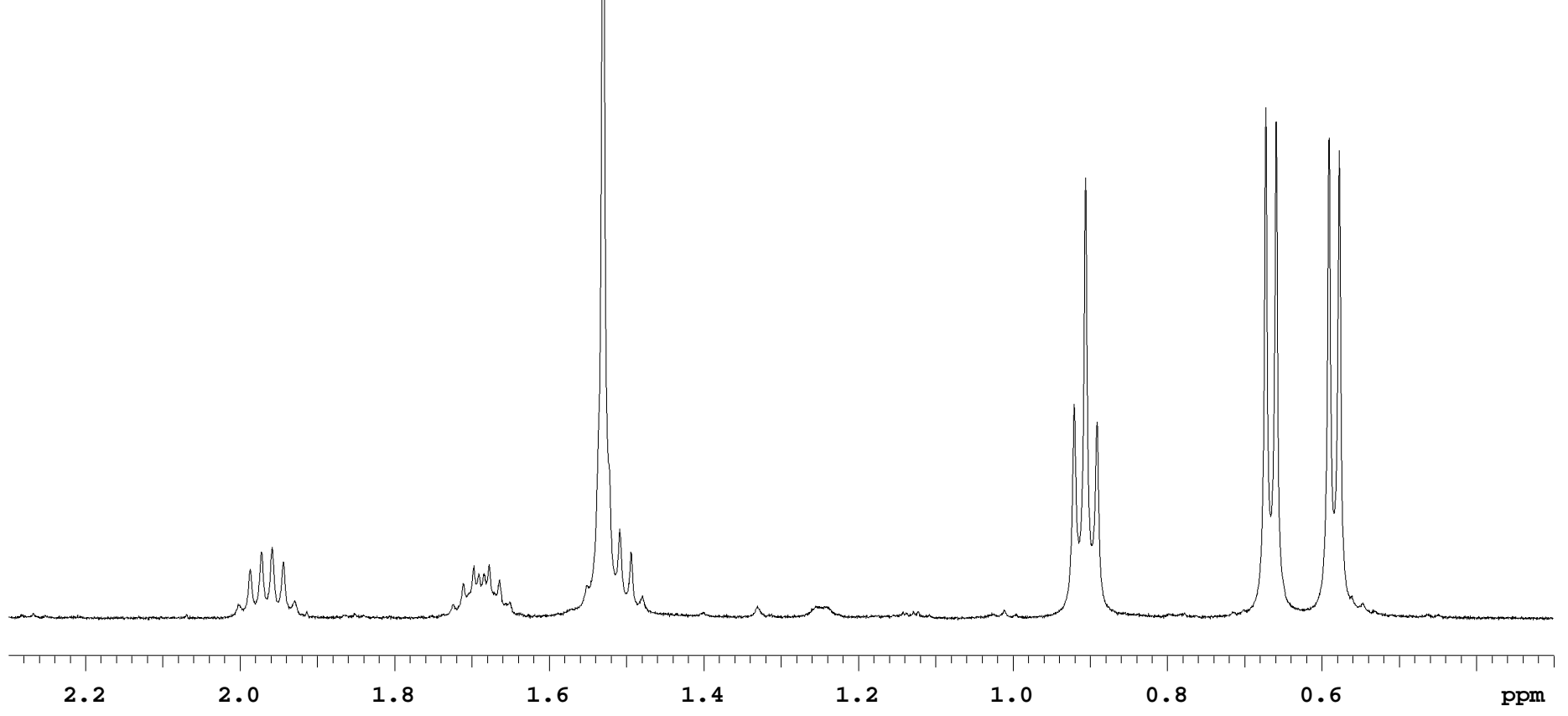




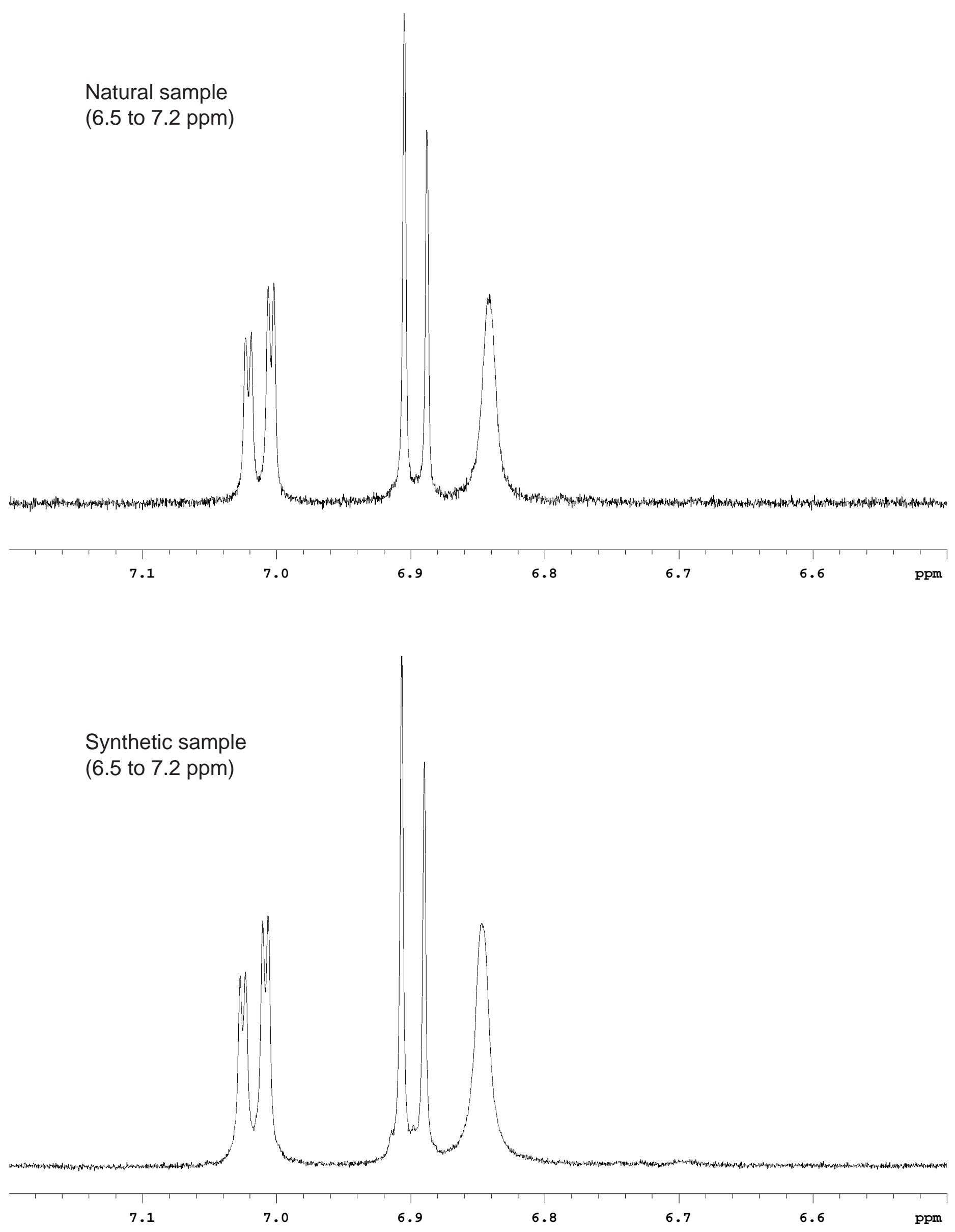


HPLC Trace of Synthetic Ustiloxin D

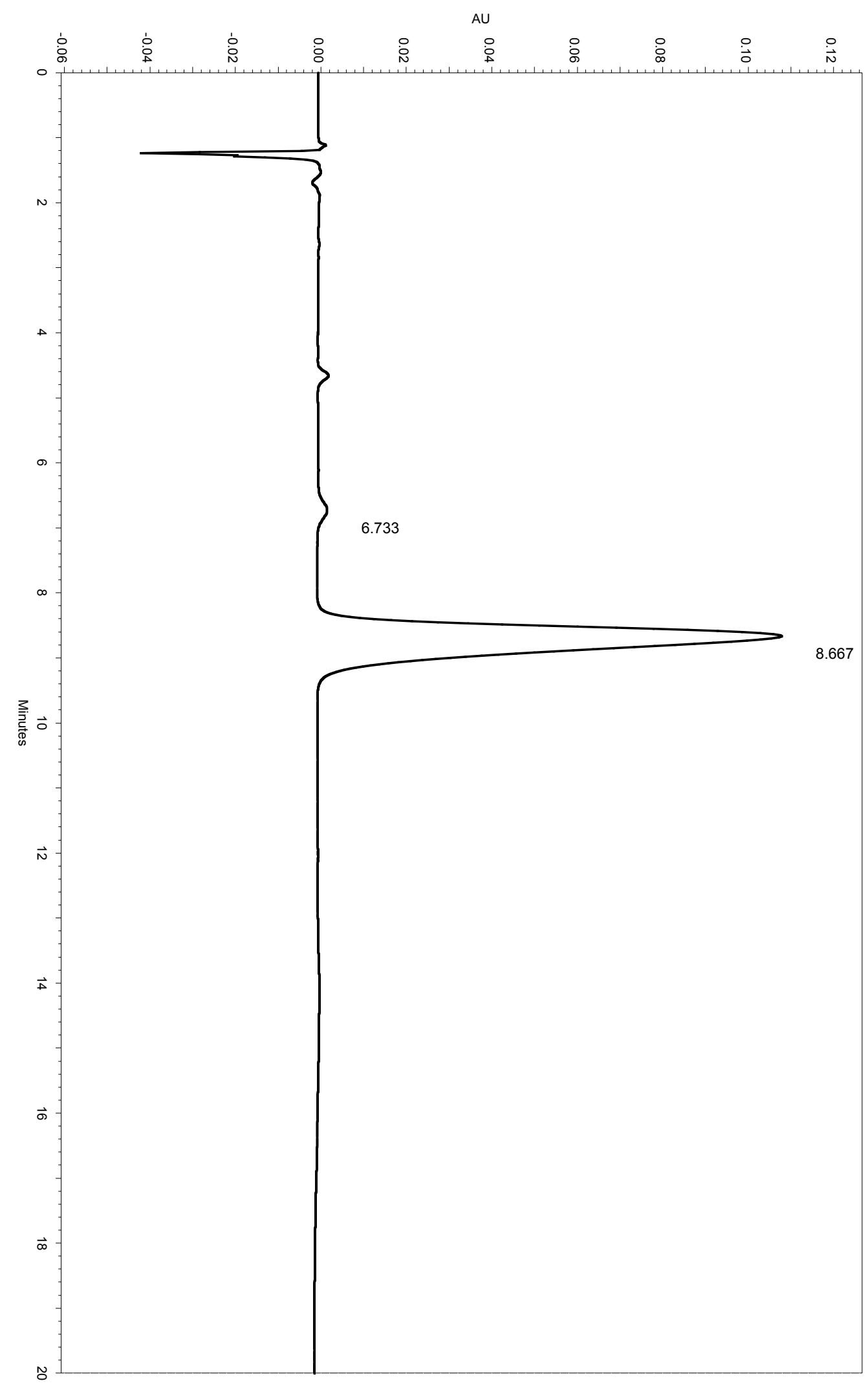

Tanaka et al. • page S10 
HPLC Trace of Natural Ustiloxin D

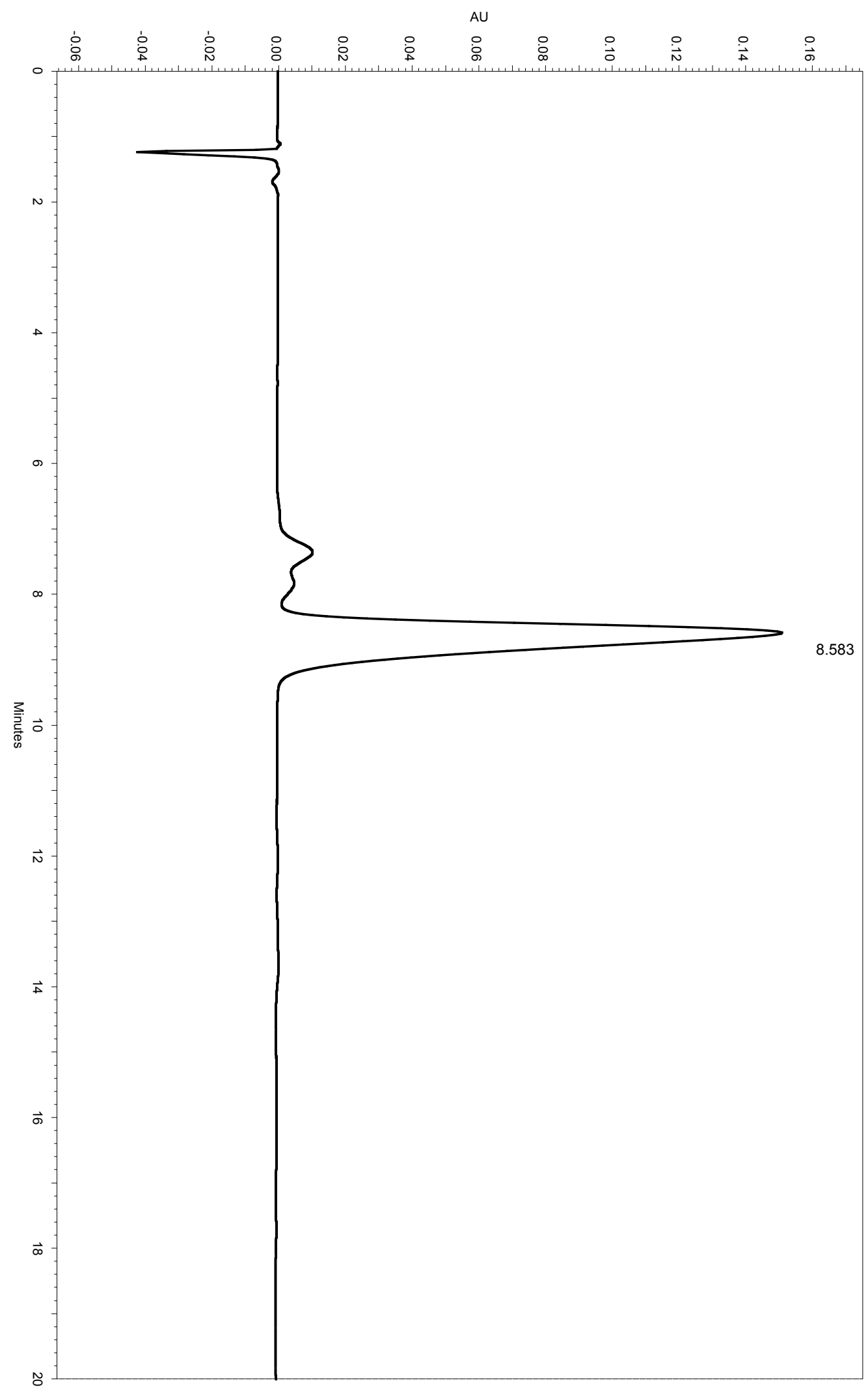

Tanaka et al. • page S11 
HPLC Trace of 1:2 Mixture of epiUstiloxin D and Synthetic Ustiloxin D

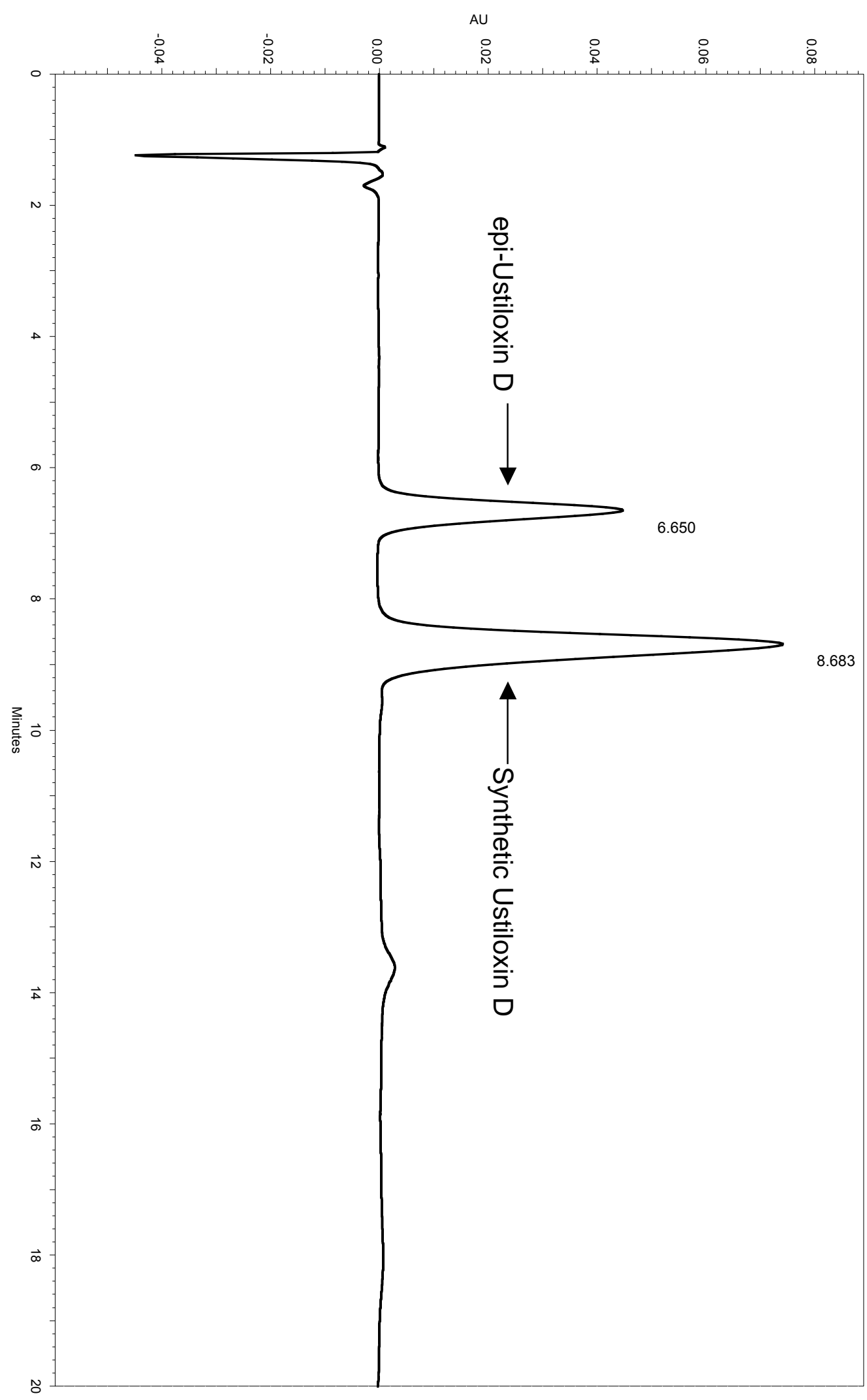

Tanaka et al. • page S12 


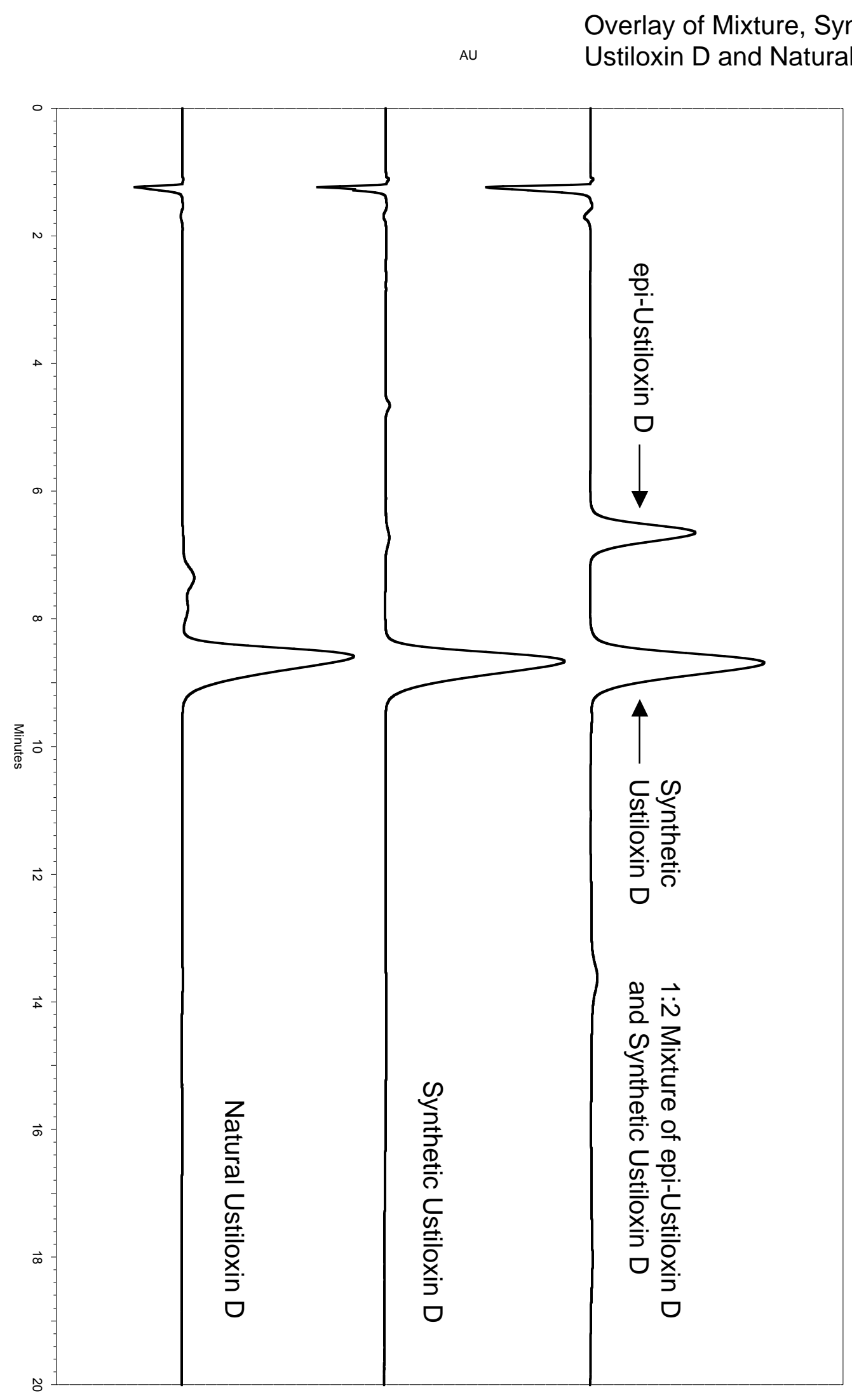

Tanaka et al. • page S13 
HPLC Trace of Co-injection of

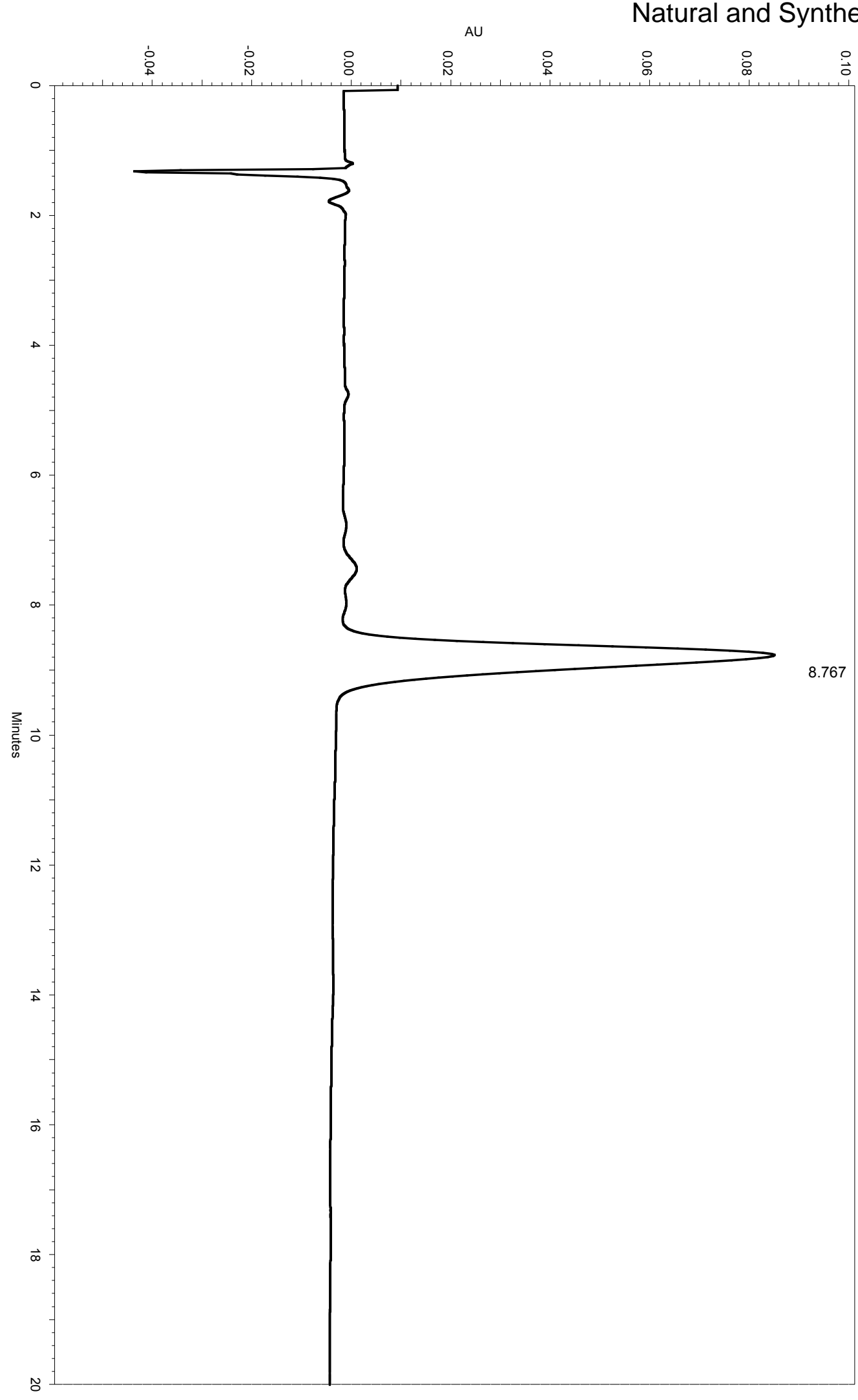

